\title{
Assessing the origins of aliphatic amines in the Murchison meteorite from their compound-specific carbon isotopic ratios and enantiomeric composition
}

\author{
José C. Aponte ${ }^{\mathrm{a}, \mathrm{b}, *}$, Jason P. Dworkin ${ }^{\mathrm{b}}$, Jamie E. Elsila ${ }^{\mathrm{b}}$ \\ ${ }^{a}$ NASA Postdoctoral Program at NASA Goddard Space Flight Center, Greenbelt, MD 20771, USA \\ ${ }^{\mathrm{b}}$ NASA Goddard Space Flight Center and Goddard Center for Astrobiology, Greenbelt, MD 20771, USA
}

Received 1 March 2014; accepted in revised form 29 June 2014; Available online 10 July 2014

\begin{abstract}
The study of meteoritic organic compounds provides a unique window into the chemical inventory of the early Solar System and prebiotic chemistry that may have been important for the origin of life on Earth. Multiple families of organic compounds have been extracted from the Murchison meteorite, which is one of the most thoroughly studied carbonaceous chondrites. The amino acids extracted from Murchison have been extensively analyzed, including measurements of nonterrestrial stable isotopic ratios and discoveries of L-enantiomeric excesses for $\alpha$-dialkyl amino acids, notably isovaline. However, although the isotopic signatures of bulk amine-containing fractions have been measured, the isotopic ratios and enantiomeric composition of individual aliphatic amines, compounds that are structurally related to amino acids, remain unknown. Here, we report a novel method for the extraction, separation, identification and quantitation of aliphatic monoamines extracted from the Murchison meteorite. Our results show a complete suite of structural isomers, with a larger concentration of methylamine and ethylamine and decreasing amine concentrations with increasing carbon number. The carbon isotopic compositions of fourteen meteoritic aliphatic monoamines were measured, with $\delta^{13} \mathrm{C}$ values ranging from $+21 \%$ to $+129 \%$, showing a decrease in ${ }^{13} \mathrm{C}$ with increasing carbon number, a relationship that may be consistent with the chain elongation mechanism under kinetic control previously proposed for meteoritic amino acids. We also found the enantiomeric composition of sec-butylamine, a structural analog to isovaline, was racemic within error, while the isovaline extracted from the same Murchison piece showed an L-enantiomeric excess of $9.7 \%$; this result suggested that processes leading to enantiomeric excess in the amino acid did not affect the amine. We used these collective data to assess the primordial synthetic origins of these meteoritic aliphatic amines and their potential linkage to meteoritic amino acids.
\end{abstract}

(C) 2014 Elsevier Ltd. All rights reserved.

\section{INTRODUCTION}

Carbonaceous chondrites represent some of the oldest and most primitive solid materials formed in the Solar System. These meteorites have recorded the history of the

* Corresponding author at: NASA Postdoctoral Program at NASA Goddard Space Flight Center, Greenbelt, MD 20771, USA. Tel.: +1 301614 6916; fax: +1 3012861683 .

E-mail address: jose.c.aponte@nasa.gov (J.C. Aponte). formation of the molecular cloud, the solar nebula, and subsequent development of the protoplanetary disc (Anders, 1989; Chyba and Sagan, 1992; Pizzarello and Shock, 2010). The Murchison meteorite is a CM2 carbonaceous chondrite that fell in southeastern Australia in 1969 and represents the most thoroughly studied carbonaceous chondrite with respect to its organic chemistry (Kvenvolden et al., 1970; Yuen and Kvenvolden, 1973; Cronin and Chang, 1993; Meierhenrich et al., 2004; Schmitt-Kopplin et al., 2010). Multiple organic classes, 
including amino acids, carboxylic acids, aliphatic amines, polycyclic aromatic hydrocarbons, nucleobases and polyols have been identified from this meteorite (e.g. Jungclaus et al., 1976; Krishnamurthy et al., 1992; Cooper et al., 2001; Martins et al., 2008; Callahan et al., 2011), providing valuable insights into the chemical inventory of the early Solar System, the primordial synthesis of organic matter, and the question of how life appeared on Earth.

One of the most intriguing questions about meteoritic organics is the origin of the L-enantiomeric excesses reported in a few chiral amino acids extracted from Murchison (Cronin and Pizzarello, 1997; Engel and Macko, 1997; Pizzarello and Cronin, 2000; Glavin and Dworkin, 2009) and other carbonaceous meteorites (Pizzarello et al., 2008, 2012; Glavin et al., 2012; Burton et al., 2013). The delivery of these enantioenriched amino acids to the early Earth may have contributed to the origin of biological homochirality (predominance of the L-enantiomer in biological amino acids), a fundamental unanswered question in origins of life. Several hypotheses have been proposed to explain the origin of the amino acid symmetry breaking found in some carbonaceous meteorites (Bonner, 1991; Greenberg, 1996; Meinert et al., 2014; Modica et al., 2014) and the subsequent amplification of their L-enantiomers (e.g. Soai et al., 1995; Klussman et al., 2006; Pizzarello and Groy, 2011; Glavin et al., 2012); however, the exact mechanisms remain unclear (Aponte et al., 2014).

Most meteoritic amino acids possess stable isotopic compositions $\left(\mathrm{D} / \mathrm{H},{ }^{13} \mathrm{C} /{ }^{12} \mathrm{C},{ }^{15} \mathrm{~N} /{ }^{14} \mathrm{~N}\right)$ that are enriched in the heavier isotopes relative to terrestrial sources, suggesting a link to formation in cold, interstellar environments. In addition, the ${ }^{13} \mathrm{C}$ isotopic composition of structurally different amino acids has been shown to differ according to the degree of aqueous and thermal alteration in various meteorite types (Martins et al., 2007; Burton et al., 2012), providing important constraints on their formation mechanisms (Epstein et al., 1987; Pizzarello et al., 1991, 2004; Elsila et al., 2012).

Meteoritic aliphatic monoamines (for simplicity we use "amines" throughout the manuscript) and amino acids share structural features such as carbon backbones and chiral centers. If these compound classes also share a synthetic history, then there should be evidence preserved in the molecular and isotopic distribution of these species. Specifically, since meteoritic branched aliphatic amines (e.g. secbutylamine) contain chiral centers similar to those found in some meteoritic amino acids (e.g. isovaline) (Scheme 1), it has been suggested that these compounds might share a common chemical origin (Pizzarello, 2002; Pizzarello et al., 2006; Hudson et al., 2009).

$\mathrm{C}_{1}$ and $\mathrm{C}_{2}$ aliphatic amines (methylamine and ethylamine) were detected in comet-exposed material from the Stardust sample return mission to comet Wild 2 and were

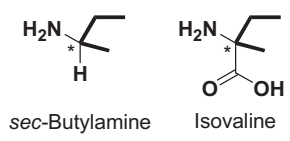

Scheme 1. Structures of sec-butylamine and isovaline (chiral center is marked with ${ }^{*}$ ). argued to be non-terrestrial in origin (Sandford et al., 2006; Glavin et al., 2008; Elsila et al., 2009), while various aliphatic amines extracted from the Murchison meteorite have been previously reported by Jungclaus et al. (1976) and Pizzarello et al. (1994). The isotopic compositions of bulk amine-containing fractions extracted from the Murchison meteorite have been measured (Pizzarello et al., 1994), but no compound-specific measurements have been reported. There is only one preliminary report in an abstract of the enantiomeric distribution of sec-butylamine in Murchison (Pizzarello, 2002). In addition, the amine content from eight Antarctic meteorites have been reported (Pizzarello and Holmes, 2009; Pizzarello et al., 2012), but the isotopic ratios and enantiomeric compositions of aliphatic amines were not determined. Without isotopic and enantiomeric information for the aliphatic amines in meteorites, it is difficult to investigate the potential synthetic relationship between amines and amino acids and to eliminate terrestrial contamination as their origin, leaving an important gap in the study of these compound families.

The objectives of the present study were: (a) to develop a novel meteorite extraction and analytical protocol for the quantification of meteoritic amines and their enantiomeric composition; (b) to measure the compound-specific $\delta^{13} \mathrm{C}$ isotopic composition of meteoritic aliphatic amines; and (c) to evaluate the potential synthetic relationships between meteoritic amines and their structurally related amino acid analogs based on their distributions and ${ }^{13} \mathrm{C}$ isotopic compositions. We also evaluated different synthetic pathways for the sec-butylamine and isovaline in light of the data, and discuss the astrophysical implications for the molecular origins of these compounds.

\section{METHODS AND MATERIALS}

\subsection{Chemicals and reagents}

Standards and reagents were purchased from Alfa Aesar or Sigma-Aldrich and used without further purification except as noted below. Ultrapure water (Millipore Direct Q3 UV, 18.2 M $\Omega, 3 \mathrm{ppb}$ total organic carbon; hereafter referred to as "water"), HPLC grade dichloromethane (DCM), semi-conductor grade $\mathrm{NaOH}$, and $6 \mathrm{M} \mathrm{HCl}$ (doubly distilled) were used. Functionalized aminopropyl and triazabicyclodecene (TBD) silica gels were obtained from SiliCycle (SiliaBond $^{\circledR}, 40-63 \mu \mathrm{m}$ particle size). (S)-(-)- $N$-(trifluoroacetyl)pyrrolidine-2-carbonyl chloride ( $S$-TPC, 97\% ee, contained trace amounts of dimethylamine) was purchased from Sigma Aldrich. All glassware, ceramics and sample handling tools were rinsed with water, wrapped in aluminum foil and heated in a muffle furnace at $500{ }^{\circ} \mathrm{C}$ in air overnight.

\subsection{Extraction of aliphatic amines}

A single chip of the Murchison carbonaceous chondrite with a visible portion of fusion crust (CM2, USNM 5451.1; total mass: $13.74 \mathrm{~g}$; extracted mass: $12.95 \mathrm{~g}$ ) was provided by the Smithsonian National Museum of Natural History, Washington, D.C. The sample, which was composed of more than 95\% inner Murchison and the rest fusion crust, 
was manually crushed and powdered using a porcelain mortar and pestle inside a positive pressure high efficiency particulate air (HEPA) filtered laminar flow hood (AirClean Systems, Inc.). The meteorite powder was separated into fourteen individual portions of approximately $900 \mathrm{mg}$ each, flame sealed inside glass ampules with $1 \mathrm{~mL}$ of $0.5 \mathrm{M} \mathrm{HCl}$, and extracted for $24 \mathrm{~h}$ at $100{ }^{\circ} \mathrm{C}$. The aqueous supernatants were removed after centrifugation, the $\mathrm{pH}$ of the supernatants measured ( $\sim 8$; various forms of carbonates and other alkaline species may explain this $\mathrm{pH}$, see De Leuw et al. (2010) and references therein), and the residual powders were rinsed twice using $0.5 \mathrm{~mL}$ of water and once using $0.5 \mathrm{~mL}$ of $6 \mathrm{M} \mathrm{HCl}$ to retain the non-volatile hydrochloride amine salts. The aqueous supernatants and rinses from the fourteen portions were combined into two fractions, which were dried under vacuum overnight. Three $\mathrm{mL}$ of water were added to each of the two dried residues, which were then centrifuged. The liquid supernatants were divided into six fractions. Each residue was also rinsed twice more ( 2 and $1 \mathrm{~mL}$ water), centrifuged, and the liquid supernatants added to the six fractions. The $\mathrm{pH}$ was continuously monitored using $\mathrm{pH}$ paper, and was observed to be acidic throughout. Next, iron hydroxides were precipitated by adding $1 \mathrm{~mL}$ of $8 \mathrm{M} \mathrm{NaOH}$ to each of the six fractions. After centrifugation, the liquid layers $(\sim 3 \mathrm{~mL}$ each) were removed and apportioned into eight vials which contained $1 \mathrm{~mL}$ of $6 \mathrm{M} \mathrm{HCl}$ each. The six vials of the precipitated iron hydroxides were additionally rinsed using $1 \mathrm{~mL}$ of water, and this water was again apportioned into the eight vials. These eight acid fractions were concentrated under vacuum, centrifuged to separate the liquid phase from the precipitated $\mathrm{NaCl}$, and combined into a single vial. This final fraction was subdivided into two equal portions; one portion was used for the analysis of free (acid-soluble) amines, while the other was subjected to acid-vapor hydrolysis using $6 \mathrm{M} \mathrm{HCl}\left(150{ }^{\circ} \mathrm{C}\right.$ for $3 \mathrm{~h}$; Glavin et al., 2006) to determine the total (free plus hydrolysable) amine content. The free and total fractions were extracted separately with $1.5 \mathrm{~mL}$ of $1 \mathrm{M} \mathrm{NaOH}$ and $1 \mathrm{~mL}$ of $\mathrm{DCM}$ followed by two portions of $0.5 \mathrm{~mL}$ of $\mathrm{DCM}$, and the organic layers were passed through a plug of baked anhydrous $\mathrm{Na}_{2} \mathrm{SO}_{4}$, which was then rinsed with $0.5 \mathrm{~mL}$ of DCM. The organic solution was stored at $-20{ }^{\circ} \mathrm{C}$ prior to derivatization. This entire extraction procedure was also carried out in parallel with a procedural blank.

\subsection{Extraction and analyses of isovaline and other amino acids}

Two pieces of the Murchison chip were separated and analyzed for amino acid content (Table S1), both to assess potential terrestrial contamination (discussed in Section 3.1) and to determine the enantiomeric composition of isovaline. The first piece $(91.1 \mathrm{mg})$ was separated from the inner part of the chip, and the second piece $(299.2 \mathrm{mg}$ ) was taken from the fusion crust; both samples were extracted and purified using published protocols (Glavin and Dworkin, 2009) for the analyses of both free and total amino acids, including isovaline. The abundance of amino acids and the enantiomeric ratio of isovaline in the meteorite extracts were determined by ultrahigh-performance liquid chromatography with fluorescence detection and time of flight mass spectrometry (LC-FD/ToF-MS) technique coupled with $o$-phthaldialdehyde/ $N$-acetyl-L-cysteine (OPA/NAC) derivatization following previously described methods (Glavin et al., 2011). The carbon isotopic composition of the amino acids in these samples was not measured because of sample size limitations.

\subsection{Derivatization of aliphatic amines}

We modified previously reported methods using enantiopure $S$-TPC as a chiral derivatization reagent (Van Bocxlaer et al., 1997; LeVasseur et al., 2008; Mohr et al., 2012). Typically, a base is needed to catalyze and achieve a complete conversion of primary and secondary amines to their corresponding amides; we used TBD bound to silica gel to obtain a complete conversion. Attempts to use other bases such as aminopropyl silica gel, hydroxides and tertiary amines produced a mixture of byproducts, incomplete reactions, or contaminated the sample with primary amines. The use of $S$-TPC as the derivatization reagent allowed resolution of a mixture of aliphatic amines, including the enantiomers of sec-butylamine, using a chiral GC column. TBD silica gel $(50 \mathrm{mg})$ and $0.1 \mathrm{M} S$-TPC in DCM $(50 \mu \mathrm{L})$ were added to the DCM extracts containing free or total amines; the slurry was stirred at room temperature for $15 \mathrm{~min}$. Next, $100 \mathrm{mg}$ of aminopropyl silica gel were added to the mixture and stirred for an additional $15 \mathrm{~min}$ to remove any excess of $S$-TPC. The slurry was passed through a glass pipette with a plug of pyrolyzed quartz wool as a filter, rinsed using $0.5 \mathrm{~mL}$ of DCM, and blown dry with nitrogen gas. Samples were dissolved in 50 or $100 \mu \mathrm{L}$ (free and total extracts respectively) of ethyl acetate for GC-MS and GC-IRMS analyses.

\subsection{Compositional and isotopic analyses}

Amine analyses were performed using gas chromatography coupled with mass spectrometry and isotope ratio mass spectrometry (GC-MS/IRMS), which provides compoundspecific identification and stable carbon isotopic ratios (Elsila et al., 2012). The gas chromatographic separation was accomplished using a Thermo Trace GC equipped with a $5 \mathrm{~m}$ base-deactivated fused silica guard column (Restek, $0.25 \mathrm{~mm}$ ID) and four $25 \mathrm{~m}$ length $\times 0.25 \mathrm{~mm}$ I.D. $\times 0.25 \mu \mathrm{m}$ film thickness Chirasil L-Val capillary columns (Agilent) connected using Press-Tight connectors (Restek). The oven program was set as follows: initial temperature was $40{ }^{\circ} \mathrm{C}$, ramped at $8{ }^{\circ} \mathrm{C} / \mathrm{min}$ to $136^{\circ} \mathrm{C}$ and held for $1 \mathrm{~min}$, ramped at $4^{\circ} \mathrm{C} / \mathrm{min}$ to $148^{\circ} \mathrm{C}$ and held for $10 \mathrm{~min}$, ramped at $2{ }^{\circ} \mathrm{C} / \mathrm{min}$ to $152^{\circ} \mathrm{C}$ and held for $22 \mathrm{~min}$, ramped at $4{ }^{\circ} \mathrm{C} / \mathrm{min}$ to $178^{\circ} \mathrm{C}$, and ramped at $10{ }^{\circ} \mathrm{C} / \mathrm{min}$ to $200{ }^{\circ} \mathrm{C}$ with a final hold of $12 \mathrm{~min}$. The carrier gas used was UHP helium (5.0 grade) at $1.8 \mathrm{~mL} / \mathrm{min}$ flow rate. Triplicate injections of derivatized amines were made in splitless mode in aliquots of $1 \mu \mathrm{L}$. Approximately $10 \%$ of the sample eluting from the GC column was directed into a Thermo DSQII electron-impact quadrupole mass spectrometer (ion source set at $200{ }^{\circ} \mathrm{C}$ and $70 \mathrm{eV}$ ). The mass 
spectrum was used to identify and quantify the meteoritic aliphatic amines by comparison to reference standards and applying a calibration curve. The $\mathrm{m} / \mathrm{z}$ of the single ion used to identify and quantify compounds 1-8, 10-14 and 18-25 was 166; compound 9 was extracted at $m / z=100$; compounds $\mathbf{1 5}$ and $\mathbf{1 6}$ at $\mathrm{m} / z=236-238$; and compound 17 at $m / z=280$. The remaining $90 \%$ of each eluting compound was directed through a Thermo GC-C III interface for oxidation of the compounds to carbon dioxide; the $\delta^{13} \mathrm{C}$ stable isotopic measurement was then made on a Thermo MAT 253 IRMS. The $\delta^{13} \mathrm{C}$ values of the eluting compounds were obtained after injection of three pulses of precalibrated $\mathrm{CO}_{2}\left(\delta^{13} \mathrm{C}=-24.23 \%\right.$ VPDB $)$ into the IRMS and computation using Thermo Isodat 2.5 software. In order to correct for the amount of carbon added by the derivatization reagent, underivatized standards were analyzed on a Costech ECS 4010 combustion elemental analyzer (EA) connected to the IRMS. The final $\delta^{13} \mathrm{C}$ values of the meteoritic amines were calculated using Eq. (1).

$$
\begin{aligned}
& \delta^{13} \mathrm{C}_{\text {sample amine }}=\left[\left(\left(n_{\text {amine }}+n_{\mathrm{d}}\right) / n_{\text {amine }}\right)\right. \\
& \left.\quad \times\left(\delta^{13} \mathrm{C}_{\text {derivatized sample amine }}-\delta^{13} \mathrm{C}_{\text {derivatized std amine }}\right)\right] \\
& +\delta^{13} \mathrm{C}_{\text {underivatized std amine }}
\end{aligned}
$$

where $n_{\text {amine }}=$ number of carbon atoms in underivatized amine and $n_{\mathrm{d}}=$ number of carbons added by derivatizing reagent. The precision (standard deviation) of the $\delta^{13} \mathrm{C}$ values was obtained using Eq. (2) (Docherty et al., 2001).

$$
\begin{aligned}
\sigma_{\text {sample amine }}^{2}= & \sigma_{\text {underivatized std }}^{2} \\
& +\left[\sigma_{\text {derivatized std }}^{2} \times\left(\left(n_{\text {amine }}+n_{\mathrm{d}}\right) / n_{\text {amine }}\right)^{2}\right] \\
& +\left[\sigma_{\text {derivatized sample }}^{2} \times\left(\left(n_{\text {amine }}+n_{\mathrm{d}}\right) / n_{\text {amine }}\right)^{2}\right]
\end{aligned}
$$

\subsection{Effects of methodology on pure amino acid and amine standards}

In order to understand whether our methodology would have a significant impact on the measured amine content, we first extracted, hydrolyzed, and derivatized a mixture of 26 amino acid standards (including isovaline and other $\mathrm{C}_{1}-\mathrm{C}_{6}$ amino acid isomers and enantiomers previously identified in the Murchison meteorite). We also evaluated the effects of the extraction, hydrolysis, and derivatization procedure on aliphatic amine standards. Enantiopure $(S)$ sec-butylamine was subjected to this protocol to determine whether significant racemization occurred. In addition, $\delta^{13} \mathrm{C}$ values were measured of both unprocessed and processed aliphatic amine standards to investigate potential isotopic fractionation effects.

\section{RESULTS AND DISCUSSION}

\subsection{Method for analysis of aliphatic amines and potential terrestrial contamination}

We developed a novel gas chromatographic method suited for the separation of a total of 25 aliphatic primary and secondary amines, including three pairs of enantiomers.
The two previous methods used to analyze aliphatic amines were developed by Jungclaus et al. (1976) who used orthophthaldialdehyde (OPA) to derivatize primary amines only; and by Pizzarello et al. (1994) who used pentafluoropropionic anhydride (PFPA) for the derivatization of primary and secondary amines; however, neither of these two protocols were suited for the enantiomeric separation of chiral amines. The conversion of the amines into their corresponding amides is presented in Scheme 2; chiral diastereomers are formed from enantiomeric amines, allowing for improved chromatographic separation on a chiral GC column and the determination of enantiomeric composition. The identities and abundances of the investigated compounds are presented in Table 1, while their structures are shown in Fig. 1.

$S$-TPC was the derivatization reagent of choice given its commercial availability, chiral nature, and low number of carbon atoms added during derivatization. However, this reagent had one minor drawback; it contained a trace amount of dimethylamine, as seen in the procedural blank (Fig. 2). The ${ }^{13} \mathrm{C}$ isotopic value of dimethylamine present in the procedural blank was not measurable given its low concentration and thus is a minor contributor to the ${ }^{13} \mathrm{C}$ isotopic value of meteoritic dimethylamine. The acid form of $S$-TPC (Fig. 2, peak R) coeluted with two chiral amines having five carbons $\left(\mathrm{C}_{5}\right)((R)$-sec-pentylamine, and (S)-3-methyl-2-butylamine) and a secondary amine (ethylpropylamine); however, we identified and quantified these compounds either by running the derivatization reactions using an excess of the amine standard over $S$-TPC (in which case the $S$-TPC-acid peak does not appear), or by using their distinctive fragmentation patterns in the mass spectrometer.

No racemization was observed when the enantiopure $(S)$-sec-butylamine standard was taken through the extraction, hydrolysis, and derivatization procedure, and $\delta^{13} \mathrm{C}$ variations were within error after aliphatic amine standards were taken through the entire analytical protocol (Table S2). Our workup procedure appears to accurately measure the original enantiomeric and isotopic composition of aliphatic amines.

Terrestrial contamination in the Murchison sample was inferred from the amino acid contents. We measured the free and total amino acid content of both the fusion crust and interior of the Murchison meteorite sample; results are shown in Table S1. The analyses indicated contamination by terrestrial amino acids, evident in the increased

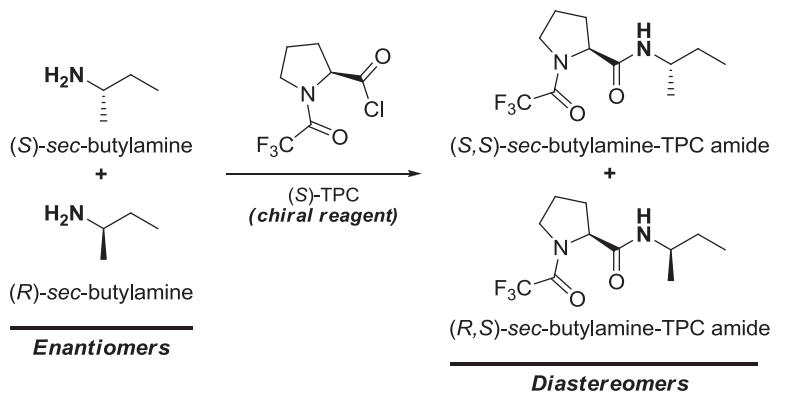

Scheme 2. Synthesis of diastereomeric sec-butylamine amides. TPC is: (S)-(-)- $N$-(Trifluoroacetyl)pyrrolidine-2-carbonyl chloride. 
Table 1

Concentrations and $\delta^{13} \mathrm{C}$ values (\% VPDB) for free and total aliphatic amines extracted from the Murchison CM2 carbonaceous chondrite.

\begin{tabular}{|c|c|c|c|c|c|}
\hline \multirow[t]{2}{*}{ Compound } & \multirow[t]{2}{*}{ Aliphatic amine ${ }^{a}$} & \multicolumn{2}{|c|}{ Abundance $(\mathrm{nmol} / \mathrm{g})^{\mathrm{b}}$} & \multicolumn{2}{|c|}{$\delta^{13} \mathrm{C}(\%)^{\mathrm{b}}$} \\
\hline & & Free & Total & Free & Total \\
\hline 1 & tert-Butylamine & $2.79 \pm 0.23$ & $2.55 \pm 0.25$ & $44 \pm 2$ & $42 \pm 1$ \\
\hline 2 & Isopropylamine & $7.24 \pm 0.75$ & $7.88 \pm 0.57$ & $42 \pm 3$ & $42 \pm 1$ \\
\hline 3 & Methylamine & $57.37 \pm 4.01$ & $85.11 \pm 7.74$ & $94 \pm 5$ & $129 \pm 7$ \\
\hline 4 & Dimethylamine & $2.45 \pm 0.60^{\mathrm{c}}$ & $2.68 \pm 0.40^{\mathrm{c}}$ & $23 \pm 4^{c}$ & $23 \pm 5^{\mathrm{c}}$ \\
\hline 5 & Ethylamine & $10.09 \pm 0.74$ & $24.13 \pm 2.80$ & $80 \pm 3$ & $80 \pm 2$ \\
\hline 6 & tert-Pentylamine & $1.92 \pm 0.37$ & $1.71 \pm 0.20$ & $34 \pm 1$ & $21 \pm 1^{\mathrm{c}}$ \\
\hline 7 & Ethylmethylamine & $1.29 \pm 0.35$ & $1.54 \pm 0.17$ & n.d. ${ }^{\mathrm{d}}$ & n.d. ${ }^{\text {d }}$ \\
\hline 8 & (R)-sec-Butylamine & $3.11 \pm 0.22$ & $5.33 \pm 0.57$ & $34 \pm 2$ & $37 \pm 2$ \\
\hline 9 & Diethylamine & $0.81 \pm 0.09$ & $0.82 \pm 0.08$ & n.d. ${ }^{\mathrm{d}}$ & n.d. ${ }^{\mathrm{d}}$ \\
\hline 10 & $(S)$-sec-Butylamine & $2.93 \pm 0.18$ & $5.03 \pm 0.57$ & $34 \pm 1$ & $35 \pm 1$ \\
\hline 11 & $n$-Propylamine & $4.33 \pm 0.30$ & $6.39 \pm 0.68$ & $35 \pm 1$ & $40 \pm 1$ \\
\hline 12 & ( $R$ )-3-Methyl-2-butylamine & $1.81 \pm 0.22$ & $2.06 \pm 0.29$ & $40 \pm 1$ & $34 \pm 2^{\mathrm{c}}$ \\
\hline 13 & Methylpropylamine & $0.73 \pm 0.25$ & $0.98 \pm 0.17$ & n.d. ${ }^{\mathrm{e}}$ & n.d. ${ }^{\mathrm{e}}$ \\
\hline 14 & Isobutylamine & $0.93 \pm 0.32$ & $1.63 \pm 0.16$ & n.d. ${ }^{\mathrm{e}}$ & n.d. ${ }^{\mathrm{e}}$ \\
\hline 15 & $(R)$-sec-Pentylamine & $1.16 \pm 0.13$ & $1.41 \pm 0.17$ & n.d. ${ }^{\mathrm{e}}$ & n.d. ${ }^{\mathrm{e}}$ \\
\hline 16 & (S)-3-Methyl-2-butylamine & $2.07 \pm 0.27$ & $2.39 \pm 0.34$ & n.d. ${ }^{\mathrm{e}}$ & n.d. ${ }^{\mathrm{e}}$ \\
\hline 17 & Ethylpropylamine & $0.08 \pm 0.00$ & $0.15 \pm 0.00$ & n.d. ${ }^{\mathrm{e}}$ & n.d. ${ }^{\mathrm{e}}$ \\
\hline 18 & 3-Pentylamine & $0.85 \pm 0.06$ & $0.93 \pm 0.11$ & $25 \pm 2$ & n.d. ${ }^{\mathrm{e}}$ \\
\hline 19 & (S)-sec-Pentylamine & $1.20 \pm 0.21$ & $1.29 \pm 0.26$ & $24 \pm 2$ & n.d. ${ }^{\mathrm{e}}$ \\
\hline 20 & $n$-Butylamine & $0.86 \pm 0.09$ & $1.05 \pm 0.12$ & $37 \pm 1$ & $43 \pm 2$ \\
\hline 21 & $(R, S)$-2-Methylbutylamine & $0.74 \pm 0.07$ & $0.73 \pm 0.07$ & $46 \pm 1$ & $45 \pm 1$ \\
\hline 22 & Isopentylamine & $0.39 \pm 0.08$ & $0.13 \pm 0.01$ & n.d. ${ }^{f}$ & n.d. ${ }^{f}$ \\
\hline 23 & $n$-Pentylamine & $0.16 \pm 0.03$ & $0.25 \pm 0.02$ & n.d. ${ }^{f}$ & n.d. ${ }^{\mathrm{f}}$ \\
\hline 24 & Pyrrolidine & $0.36 \pm 0.12$ & $0.47 \pm 0.05$ & n.d. ${ }^{f}$ & n.d. ${ }^{f}$ \\
\hline 25 & $n$-Hexylamine & $0.13 \pm 0.04$ & $0.20 \pm 0.02$ & n.d. ${ }^{f}$ & n.d. ${ }^{f}$ \\
\hline
\end{tabular}

${ }^{\text {a }}$ Compounds identified by comparison with elution time and mass spectra of standards.

${ }^{b}$ Values are the average of three measurements; errors shown are standard deviations.

${ }^{c}$ Includes small contribution from dimethylamine, phthalate or $S$-TPC acid.

${ }^{\mathrm{d}}$ Value could not be determined due to coelution or poor peak resolution.

${ }^{\text {e }}$ Value could not be determined due to coelution with $S$-TPC acid.

${ }^{\mathrm{f}}$ Value could not be determined due to coeluting compounds and limited amount of sample.

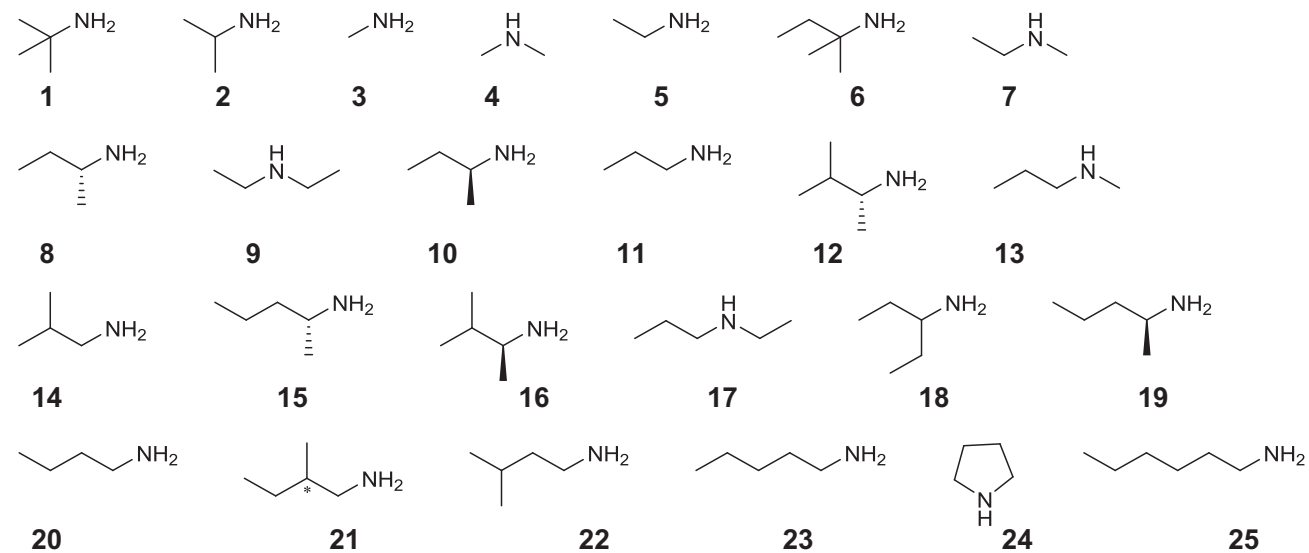

Fig. 1. Structures of aliphatic amines listed in Table 1. Unresolved chiral center marked with ${ }^{*}$

$\mathrm{L} / \mathrm{D}$ ratios of proteinogenic amino acids such as alanine and valine in the fusion crust. The interior, however, contained racemic alanine and valine, indicating that the bulk of the sample did not suffer from terrestrial contamination. Furthermore, we also noticed an increase in the amounts of isovaline from the interior chip to the fusion crust piece without changes in its L/D enantiomeric ratio. Experiments aimed at understanding mechanisms leading to the potential concentration of amino acids in the fusion crust that are beyond the scope of this investigation might be required to fully understand this observation.

In order to investigate the potential contribution of amines by the decomposition of amino acids, we took a mixture of $200 \mu \mathrm{mol}$ of each of 26 amino acids and 

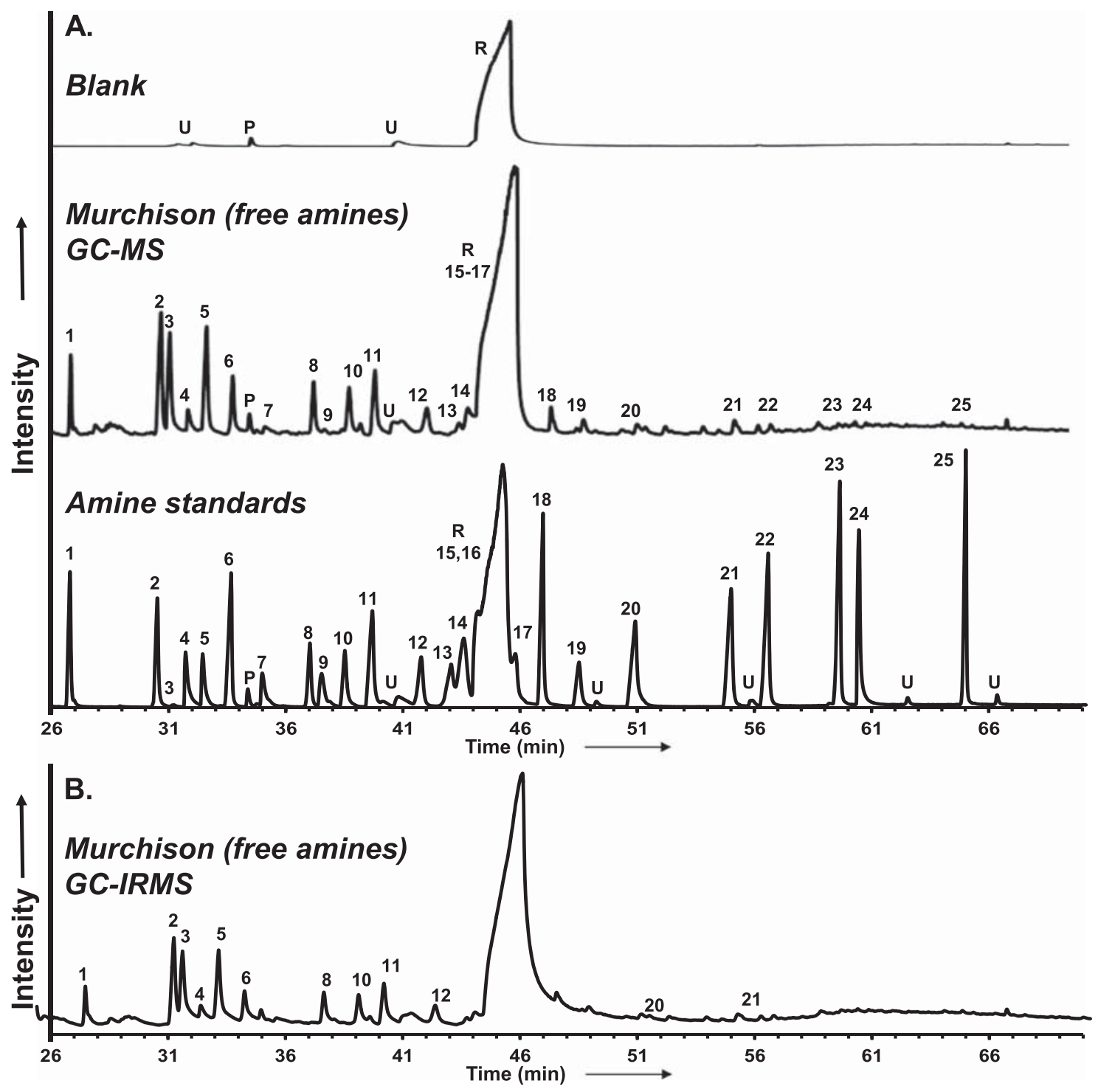

Fig. 2. Panel A shows a positive electron impact GC-MS chromatogram (26.0-70.7 min region) of procedural blank, free amines extracted from the Murchison meteorite and commercially available amine standards. Similar chromatograms were obtained for the total extract of Murchison. Panel B shows the GC-IRMS chromatogram obtained at $m / z=44\left({ }^{12} \mathrm{CO}_{2}\right.$ peak $)$ obtained and measured during carbon compound-specific isotope analysis. The identities of the peaks and structures are presented in Table 1 and Fig. 1, respectively. U: unknown compound, P: phthalate, R: reagent ( $S$-TPC acid).

subjected it to the complete analytical protocol (extraction, hydrolysis and derivatization); we observed the production of isopropylamine and isobutylamine in trace amounts. Isopropylamine could be formed by decarboxylation of $\alpha$-aminoisobutyric acid and $\beta$-aminobutyric acid; while isobutylamine could be derived from the decomposition of valine, 3-amino-2,2-dimethyl-propanoic acid, and 4amino-3-methylbutanoic acid.

Since the potential meteoritic amino acid precursors of isopropylamine and isobutylamine we extracted from the Murchison sample were at minimum 1333 (inner) and 42 (crust) times lower than those in this experiment, the contribution of any amines produced during the workup are well below our quantitation and isotopic measurement errors so they would not interfere with our measurements of indigenous meteoritic aliphatic amines.

\subsection{Distribution and quantification of aliphatic amines in the Murchison meteorite}

Fig. 2 presents the total ion chromatogram of a procedural blank, a representative injection from the Murchison meteorite through GC-MS and GC-IRMS, and the mixture of amine standards, illustrating the application of our analytical protocol to a meteorite extract. We analyzed both the free and total amine content of the Murchison meteorite sample. The "free" extract contains those soluble or chemically unbound amines that are readily extracted in 
the aqueous $0.5 \mathrm{M} \mathrm{HCl}$ fraction, while the "total" extract contains both $0.5 \mathrm{M} \mathrm{HCl}$ soluble amines and those bound that are released after $6 \mathrm{M} \mathrm{HCl}$ vapor hydrolysis. Fig. 3 compares the amine content of the two extracts.

We found that after hydrolysis methylamine and ethylamine (48\% and 139\%, respectively) and other primary amines having linear aliphatic chains showed a significant increase in their concentrations. Any increase in the abundance of branched and secondary amines were within our analytical uncertainties (Table 1, Fig. 3). The observed increase in the abundance of linear amines is in agreement with Jungclaus et al. (1976) who reported that the amount of some meteoritic aliphatic amines in Murchison increased more than $100 \%$ after hydrolysis in liquid $6 \mathrm{M} \mathrm{HCl}$. Hydrolysis liberates amines from bound precursor forms, for example from amides in their free-soluble form and those bound to small size particles of insoluble organic matter.

We identified a total of 25 meteoritic amines in the Murchison meteorite, including all amines previously reported by Jungclaus et al. (1976), as well as all the amines reported by Pizzarello et al. (1994) with the exception of butylmethylamine, for which we lacked the reference standard. In addition, we identified the compounds pyrrolidine (a cyclic secondary amine) and $n$-hexylamine, which to our knowledge have not been previously reported in Murchison or any other meteorite. Our results show a complete suite of

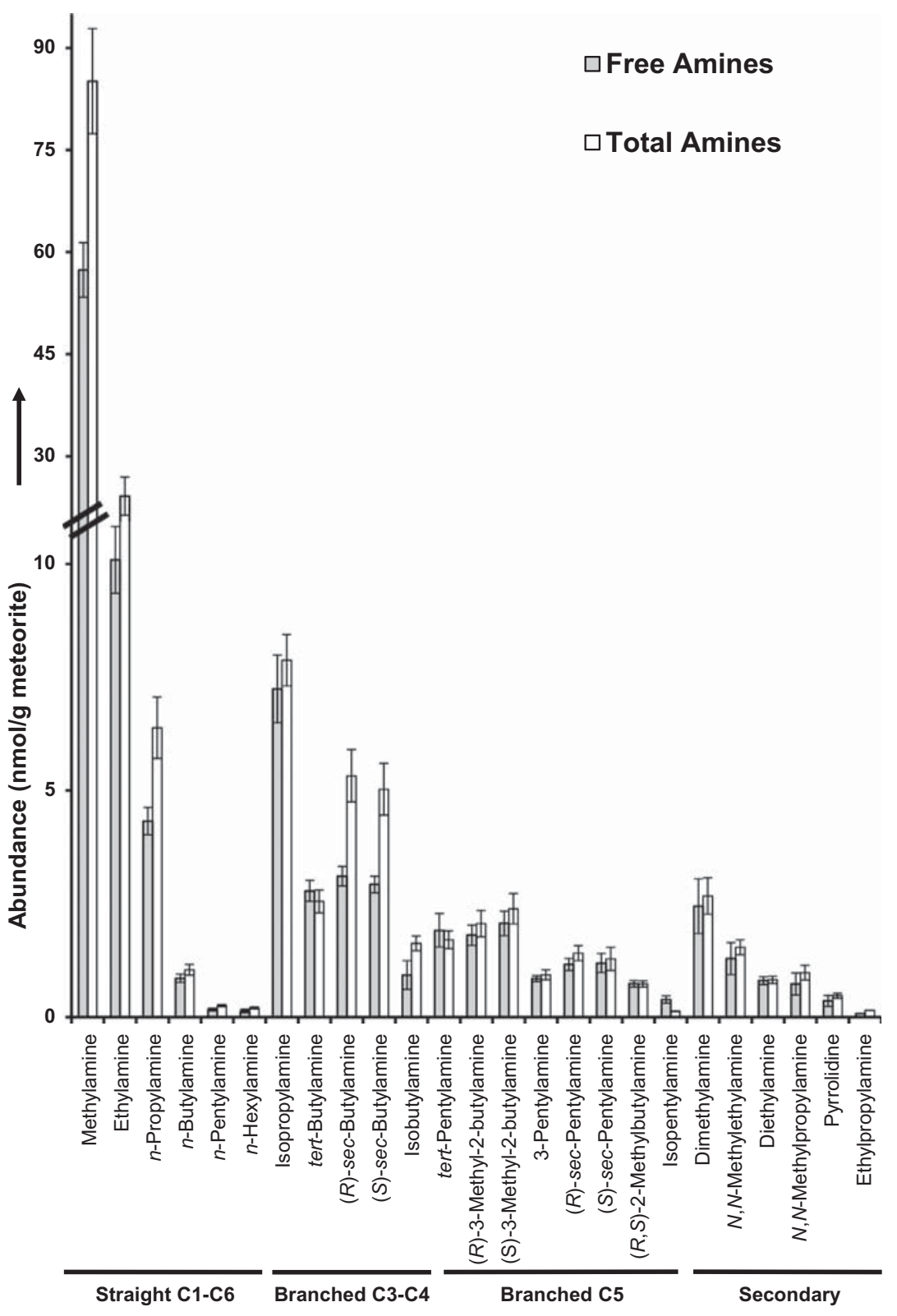

Fig. 3. Abundances of 25 free (acid-soluble) and total (free plus those released by $6 \mathrm{M} \mathrm{HCl}$-vapor hydrolysis) amines extracted from the Murchison CM2 carbonaceous chondrite. Compounds were plotted in order of increasing number of carbon atoms and grouped according to their molecular structures. 
$\mathrm{C}_{1}-\mathrm{C}_{4}$ aliphatic amines (with higher concentration of methylamine and ethylamine), and a trend of decreasing abundance of primary and secondary aliphatic amines with increasing number of carbons (Table 1, Fig. 3). The concentrations of free and total amines were 106 and $157 \mathrm{nmol} / \mathrm{g}$ of meteorite respectively; methylamine was the most abundant amine, constituting $54 \%$ of the concentration of free amines and total amines. Secondary amines were lower in concentration than primary amines; they represented 5\% and $6 \%$ of extracted free and total amines respectively. The overall high concentration of methylamine and the low abundance of secondary amines are in agreement with previous measurements of Murchison (Jungclaus et al., 1976; Pizzarello et al., 1994).

A comparison of individual compound abundances indicates that the amount of primary amines we detected were 1.2-2.8 times lower than those found by Jungclaus et al. (1976) but up to 2.3 times higher than the abundances reported by Pizzarello et al. (1994). Differences in concentrations could be attributed to different extraction and derivatization methodologies; extraction efficiencies may vary, and $S$-TPC as well as the other derivatization reagents used previously may react at different rates with amines depending on their steric hindrance and steric shielding, leading to enhancement or suppression of relative abundances in a mixture. However, we did not observe a direct relationship between amine structure and comparisons of measured abundances with previous reports. For example, we detected 1.7 and 2. 8 times less $n$-propylamine and isopropylamine than Jungclaus et al. (1976), but similar amounts of the more hindered tert-butylamine; analogously, we obtained higher amounts of sec-butylamine than Pizzarello et al. (1994), but similar amounts of less hindered propylamine and isopropylamine. Therefore, we consider that the differences in both absolute and relative concentrations could be due to heterogeneity within the Murchison meteorite, as has been reported previously for other organic compounds (Cronin and Pizzarello, 1983; Krishnamurthy et al., 1992; Pizzarello et al., 2003). Nevertheless, the complete structural diversity and trend of lowering abundance with increasing molecular weight is similar to that seen in other compound classes such as meteoritic aliphatic amino acids and carboxylic acids (Cronin and Pizzarello, 1983, 1986; Huang et al., 2005; Aponte et al., 2011). These similarities in compound distributions may be indicative of the common origins and development throughout the Solar System.

\subsection{Compound-specific $\delta^{13} \mathrm{C}$ analysis of meteoritic aliphatic amines}

Table 1 and Fig. 4 present the $\delta^{13} \mathrm{C}$ values we measured for the amines extracted from the Murchison meteorite; all measured compounds were substantially enriched in ${ }^{13} \mathrm{C}$ relative to biogenic organics, strongly supporting an extraterrestrial origin (Scott et al., 2006; Larsen et al., 2009). Given the presence of terrestrial amino acid contamination in the fusion crust of this sample (Section 3.1) it is reasonable that some terrestrial amines are also present, thus $\delta^{13} \mathrm{C}$ values presented here should be considered as a lower limit for extraterrestrial Murchison amines.

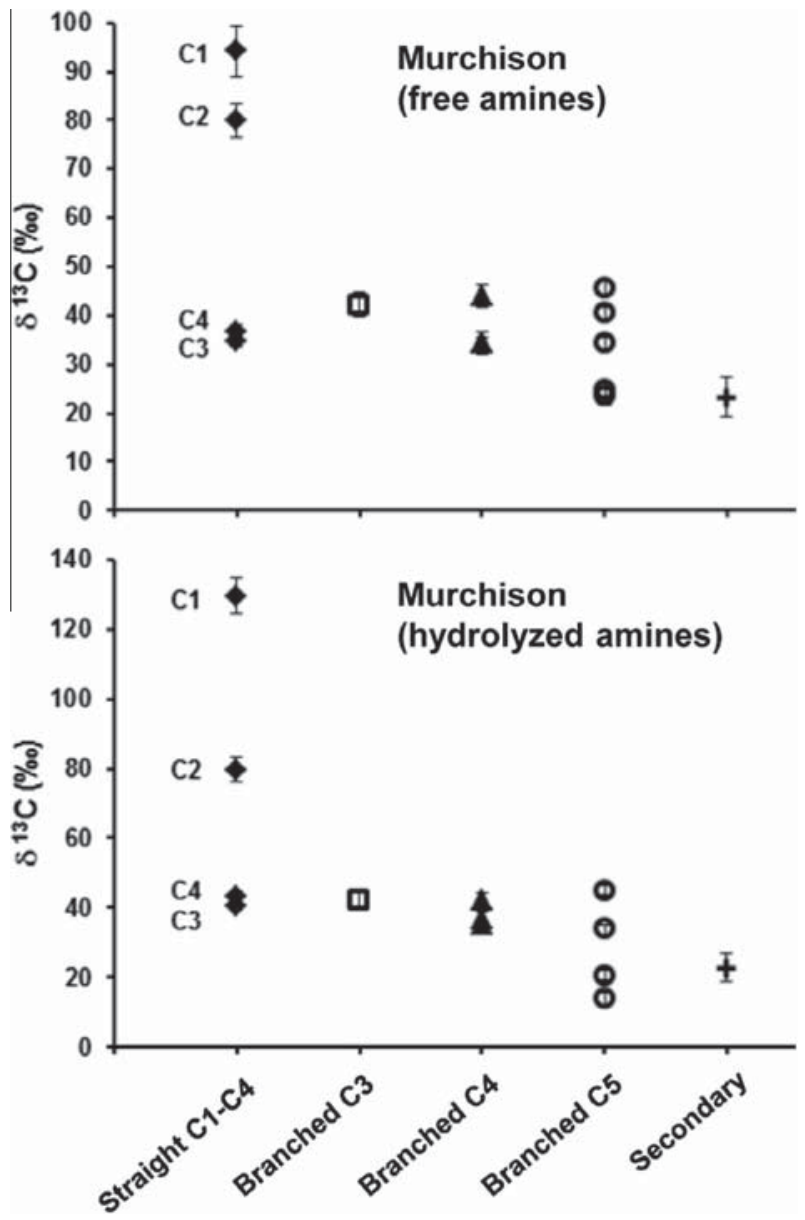

Fig. 4. $\delta^{13} \mathrm{C}$ isotopic data for the aliphatic amines extracted from the Murchison meteorite. The compounds were grouped by increasing number of carbon atoms and according to their molecular structures.

The compound-specific $\delta^{13} \mathrm{C}$ values ranged from $+23 \%$ to $+94 \%$ for free amines, and from $+21 \%$ to $+129 \%$ for total amines, with methylamine possessing the highest $\delta^{13} \mathrm{C}$ values in both (Table 1$)$. These enriched carbon isotopic values constrain the primordial synthesis of aliphatic amines or their precursors to cold interstellar environments. The only previous assessment of the carbon isotopic composition of meteoritic amines was reported by Pizzarello et al. (1994). In that report, the total hydrolyzed amine extract exhibited a $\delta^{13} \mathrm{C}$ of $+22 \%$; no compound-specific measurements were made. As with the previously discussed variations in amine concentrations, there are several possibilities for the relative enrichment of our compound-specific measurements compared to the previous bulk measurement. First, amines may have been measured with different efficiencies in the two studies. Second, the differences may reflect the inherent variability of the organic composition in different fragments of the Murchison meteorite. Third, the bulk extract previously measured may have contained organic bases other than primary and secondary aliphatic amines. It is possible that the workup by Pizzarello et al. (1994) also included tertiary amines, nitrogen heterocycles, 
etc. and may have lighter carbon isotopic compositions or biogenic sources and thus lowered the $\delta^{13} \mathrm{C}$ value of the bulk basic extract.

Table 1 compares the carbon isotopic ratios of the free and total amines. For most compounds, there was no significant difference between these two fractions. The methylamine, however, showed a significant enrichment in ${ }^{13} \mathrm{C}$ in the hydrolyzed fraction compared to the unhydrolyzed fraction. Slight ${ }^{13} \mathrm{C}$ enrichment was observed in $n$-propylamine and $n$-butylamine; based on the analysis of standards (Table S2), it is unlikely that the hydrolysis method causes isotopic fractionation of amines. These results suggest that the increase in ${ }^{13} \mathrm{C}$ content of these amines in the hydrolyzed extract is due to the addition of "bound" amines released after vapor hydrolysis. These bound amines could include amides or other precursors present in the initial aqueous extract. This observation is supported by the increase in the abundance of these molecules after hydrolysis (Table 1).

The amines tert-pentylamine and $(R)$-3-methyl-2-butylamine showed a slight-to-moderate depletion in ${ }^{13} \mathrm{C}$ in the total fraction compared to the free fraction. The depletion of the ${ }^{13} \mathrm{C}$ content after hydrolysis is likely due to the addition of ${ }^{12} \mathrm{C}$ enriched molecules from terrestrial chromatographic interference. The $(R)$-3-methyl-2-butylamine peak contained a small contribution from $S$-TPC acid, and tert-pentylamine partially coeluted with an unidentified phthalate. The triplicate analyses of the free fraction had better chromatography, and thus these compounds were better resolved from the contaminants.

We compared the carbon isotopic values for the amines with structural features such as number of carbons and whether they were linear or branched-chain (Fig. 4). For the small-chain linear amines, we see a decrease in $\delta^{13} \mathrm{C}$ value with an increase in carbon number, beginning with methylamine $(+129 \%$ for the total extract, $\mathrm{C} 1)$ and continuing through ethylamine $\delta^{13} \mathrm{C}(+80 \%, \mathrm{C} 2)$ to $n$-propylamine (C3) and $n$-butylamine (C4) (35\% and $37 \%$, respectively). Among branched C3-, C4- and C5-amines, there is a significant variability in their $\delta^{13} \mathrm{C}$ values, ranging from $+24 \%$ to $+46 \%$ for free amines and from $+21 \%$ to $+45 \%$ for the total amines fraction; however, an overall depletion of ${ }^{13} \mathrm{C}$ with increasing carbon number can be observed (Fig. 4).

The observed trends of decreasing $\delta^{13} \mathrm{C}$ with increasing carbon number suggests the synthesis of larger molecules by the gradual addition of more reactive ${ }^{12} \mathrm{C}$ atoms under kinetic control as has been proposed for other meteoritic compounds (Yuen et al., 1984), and as has been observed for amino acids in a variety of carbonaceous chondrites including Murchison (Engel et al., 1990; Pizzarello and Cooper, 2001; Ehrenfreund et al., 2001a; Pizzarello et al., 2004, 2008; Martins et al., 2007; Elsila et al., 2012). One interesting observation is the sharp carbon isotopic difference observed between dimethylamine and methylamine $(+23 \%$ and $+129 \%$, respectively). This large difference is unlikely to be solely caused by the trace amounts of dimethylamine present in the $S$-TPC reagent (see Section 3.1). As has been argued with chain elongation, it is plausible that the ${ }^{13} \mathrm{C}$-enriched methylamine remained after the slightly more reactive ${ }^{12} \mathrm{C}$-methylamine and ${ }^{12} \mathrm{C}$-methyl ions were consumed to produce dimethylamine.

\subsection{Comparison of $\delta^{13} \mathrm{C}$ values between meteoritic amino acids and amines}

To explore the potential parent-daughter synthetic relationship between meteoritic amines and amino acids, Table 2 compares the $\delta^{13} \mathrm{C}$ values of the aliphatic amines measured in this study with the $\delta^{13} \mathrm{C}$ values for analogous Murchison amino acids (Engel et al., 1990; Pizzarello et al., 2004; Elsila et al., 2012). Our data show a large ${ }^{13} \mathrm{C}$ isotopic difference between the $\mathrm{C}_{1}$ and $\mathrm{C}_{2}$ primary amines (methylamine and ethylamine) and their amino acid analogs (glycine and $\alpha$ - or $\beta$-alanine). The straight-chain $\mathrm{C}_{3}$ amine (n-propylamine), shows slight ${ }^{13} \mathrm{C}$ enrichment over one of its corresponding amino acid analogs $(\alpha$-amino$n$-butyric acid) $\left(\delta^{13} \mathrm{C}\right.$ values for $\beta$-aminoisobutyric acid and $\gamma$-amino- $n$-butyric acid are not available for Murchison). In contrast, the $\delta^{13} \mathrm{C}$ values observed for branched $\mathrm{C}_{3}$ and $\mathrm{C}_{4}$ amines (isopropylamine and sec-butylamine) and their corresponding amino acids $(\alpha$-aminoisobutyric acid and isovaline) are the same within analytical uncertainties $\left( \pm 4-5 \%\right.$, Table 2). Again, no $\delta^{13} \mathrm{C}$ values are available for the minor $\mathrm{C}_{5}$ amino acids; isobutylamine coelution with $S$-TCP acid precluded to measure its $\delta^{13} \mathrm{C}$ for a comparison with valine.

To understand the high isotopic difference between methylamine and ethylamine and their amino acid analogs, we first evaluated various possible sources of fractionation. Previously measured D- and L-alanine $\delta^{13} \mathrm{C}$ values were identical within error (Table 2; Engel et al., 1990; Pizzarello et al., 2004; Elsila et al., 2012), indicating a lack of contamination from terrestrial biological L-alanine. This observation is inconsistent with the large amounts of terrestrial amino acids that would be needed to account for a $>40 \%$ difference between methylamine and ethylamine and their analogous amino acids; therefore, we disregard terrestrial contamination as the cause of the large $\delta^{13} \mathrm{C}$ difference found between these compound classes. Meteorite sample variability is also unlikely to account for such large isotopic differences; although isotopic ratios may vary somewhat throughout a meteorite, glycine and alanine $\delta^{13} \mathrm{C}$ values have been shown to be relatively consistent across several different CM2 chondrites (Elsila et al., 2012), and Table 2 shows that the range of amino acid $\delta^{13} \mathrm{C}$ values measured in separate samples of the Murchison meteorite is smaller than the difference between the $\mathrm{C}_{1}$ and $\mathrm{C}_{2}$ amino acids and their corresponding amines. Isotopic fractionation during extraction and workup also seems unlikely, given that our experiments with aliphatic amine standards showed no variation in their ${ }^{13} \mathrm{C}$ content after extraction and workup (Table S2). The ${ }^{13} \mathrm{C}$ enrichment could be produced by the isotopic fractionation during loss of the more volatile compounds such as methylamine and ethylamine either during their residence on the parent body or since the collection of the Murchison meteorite. If amines and amino acids had the same $\delta^{13} \mathrm{C}$ initially, but the amines preferentially lost the lighter fraction, they would be more enriched in ${ }^{13} \mathrm{C}$; this process of fractionation 
Table 2

Comparison of the $\delta^{13} \mathrm{C}(\%$ VPDB) values of structurally analogous amino acids (previous studies) and aliphatic amines (this study) from the Murchison CM2 carbonaceous chondrite.

\begin{tabular}{llllr}
\hline Murchison & & & & \\
\hline Amino acid & $\delta^{13} \mathrm{C}$ & Aliphatic amine & Free $\delta^{13} \mathrm{C}$ & Total $\delta^{13} \mathrm{C}$ \\
\hline Glycine & $22^{\mathrm{a}}, 41^{\mathrm{b}}, 13^{\mathrm{c}}$ & Methylamine & \\
D-Alanine & $30^{\mathrm{a}}, 52^{\mathrm{b}}, 38^{\mathrm{c}}$ & Ethylamine & 84 & 80 \\
L-Alanine & $27^{\mathrm{a}}, 38^{\mathrm{b}}, 40^{\mathrm{c}}$ & & & \\
$\beta$-alanine & $5^{\mathrm{b}}, 10^{\mathrm{c}}$ & & 35 & \\
D- $\alpha$-ABA & $29^{\mathrm{b}}, 18^{\mathrm{c}}$ & $n$-Propylamine & 42 & 40 \\
$\alpha$-AIB & $5^{\mathrm{a}}, 43^{\mathrm{b}}, 38^{\mathrm{c}}$ & Isopropylamine & 34 \\
D,L-Isovaline & $17^{\mathrm{a}}, 22^{\mathrm{b}}, 39^{\mathrm{c}}$ & $($ R)-sec-Butylamine & 34 & 34 \\
\hline
\end{tabular}

ABA: aminobutyric acid; AIB: aminoisobutyric acid.

${ }^{a}$ Engel et al. (1990).

b Pizzarello et al. (2004).

${ }^{\mathrm{c}}$ Elsila et al. (2012).

would also affect their abundance, indicating that the original concentrations of methylamine and ethylamine would have been even higher than those we observed (Table 1), and the contribution of these two compounds to the total amine content in Murchison would have exceeded the $70 \%$ we report. Alternatively, a direct explanation for the isotopic differences and similarities found between amines and amino acids would suggest that $C_{1}$ and $C_{2}$ primary amines and their amino acid analogs were formed by distinct synthetic routes, while $\mathrm{C}_{3}$ and $\mathrm{C}_{4}$ amines and their amino acid analogs were formed by similar synthetic processes.

Methylamine can be produced by reductive amination of carbon monoxide (CO) or hydrogenation of hydrogen cyanide $(\mathrm{HCN})$; these two distinct synthetic routes can occur in gas phase, ice grains, or inside the parent body (Tielens and Hagen, 1982; Charnley et al., 2004; Theule et al., 2011; Elsila et al., 2012). These precursor molecules could then react with ${ }^{12} \mathrm{C}$-methylamine and ${ }^{12} \mathrm{CO}$ in order to produce ethylamine, glycine and other amino acid precursors such as formaldehyde and formic acid, leaving ${ }^{13} \mathrm{C}$-methylamine unreacted and resulting in products that decrease in ${ }^{13} \mathrm{C}$ content with increasing chain length and complexity. The observed isotopic differences suggest that amines and their analogous amino acids were formed from precursor molecules that amplify isotopic fractionation through a chain elongation mechanism (Yuen et al., 1984).

Elsila et al. (2012) proposed that the observed ${ }^{13} \mathrm{C}$-depletion with increasing chain length for $\alpha-\mathrm{H}, \alpha$-amino acids may result from the synthesis of these compounds from aldehydes and ketones synthesized from fractionated ${ }^{13} \mathrm{C}$ enriched $\mathrm{CO}$, and ${ }^{13} \mathrm{C}$-depleted $\mathrm{HCN}$ and alkyl groups. Under this mechanism, the carboxyl carbon derives from hydrolysis of the ${ }^{13} \mathrm{C}$-depleted HCN. This suggests that the systematic loss of the carboxylic moiety from $\alpha-\mathrm{H}$, $\alpha$-amino acids would result in aliphatic amines that are more ${ }^{13} \mathrm{C}$-enriched relative to the precursor amino acid. These observations are in agreement with our $\delta^{13} \mathrm{C}$ measurements (Table 2), suggesting that at least a fraction of methylamine and ethylamine could be decarboxylation products of glycine, and $\alpha$ - and $\beta$-alanine. Further analyses of the isotopic composition of meteoritic amines present in carbonaceous meteorites that experienced varying degrees of aqueous and thermal alterations are needed to understand their abiotic origins.

\subsection{Comparison of sec-butylamine and isovaline and potential synthetic relationship}

sec-Butylamine is of particular interest because it is structurally analogous to the amino acid isovaline (Scheme 1), which has been observed to possess a non-terrestrial L-enantiomeric excess in Murchison and a few other carbonaceous chondrites (Cronin and Pizzarello, 1997; Pizzarello et al., 2003; Glavin and Dworkin, 2009; Glavin et al., 2011; Burton et al., 2013). The two compounds contain identical aliphatic chains; their structural difference is the $\alpha$-carbonyl group present on the amino acid, which is replaced with an $\alpha-\mathrm{H}$ on the aliphatic amine. In comparing the amine and amino acid data, we observed two potential connections between these molecules. First, $(R)$ - and $(S)$ sec-butylamine were more abundant than other $\mathrm{C}_{4}$ amine isomers such as $n$-butylamine and isobutylamine. This predominance of sec-butylamine is similar to the higher relative abundance of isovaline over other $\mathrm{C}_{5}$ amino acids extracted from the Murchison meteorite (Cronin et al., 1985; Ehrenfreund et al., 2001a; Glavin and Dworkin, 2009; Glavin et al., 2011). Second, the $\delta^{13} \mathrm{C}$ value for secbutylamine measured in this study falls within the range of values previously measured for D,L-isovaline (Engel et al., 1990; Pizzarello et al., 2004; Elsila et al., 2012). Given their structural similarity, their higher abundance in relation to their constitutional isomers, and their similar carbon isotopic compositions, it has been postulated that there may be a potential synthetic relationship between sec-butylamine and isovaline (Pizzarello et al., 1994; Hudson et al. 2009).

Fig. 5 presents the gas chromatogram of $(R)$ - and $(S)$ sec-butylamine (compounds 8 and 10) monitored at $\mathrm{m} /$ $z=166.0 \pm 0.5$ and the mass spectra of these peaks in the Murchison extract compared to a standard. As seen in Table 1, the measured concentrations of $(R)$ - and 


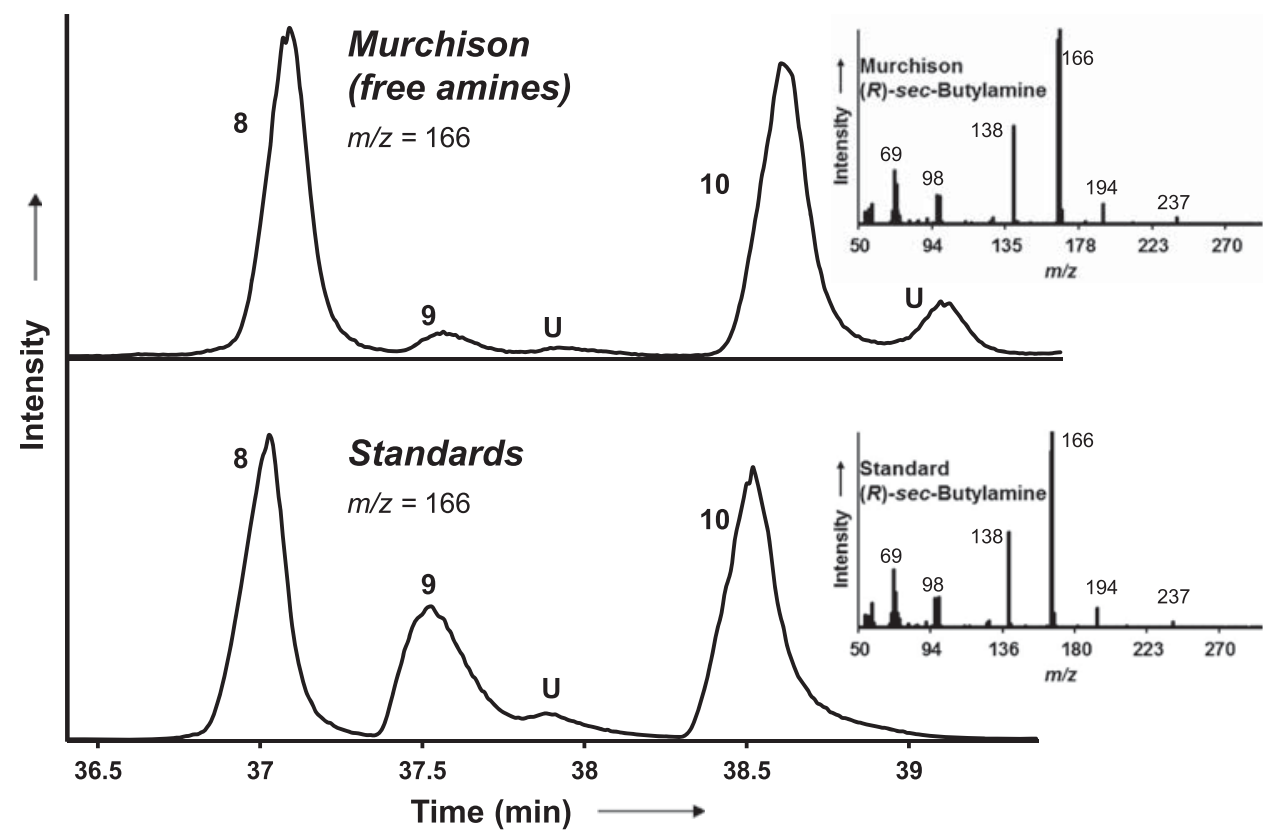

Fig. 5. Positive electron impact GC-MS chromatogram (36.5-40 min region, $m / z=166 \pm 0.5)$ of $(S)$-TPC-derivatized $(R)$ - and $(S)$-sec-butylamine (compounds 8 and 10). Similar chromatograms were obtained for the total extract of Murchison. The inserts show the simultaneously collected mass spectral fragmentation pattern for peak 8 and the standard $(R)$-sec-butylamine, with similar mass fragmentation patterns observed, indicating no coeluting peaks. U: unknown compound.

$(S)$-sec-butylamine were equal within experimental error, indicating a racemic mix of these compounds. This is the first full report of the separation and quantitation of these compounds in the Murchison meteorite. A preliminary examination of the enantiomeric composition of sec-butylamine extracted from one piece of the Murchison meteorite was previously reported in an abstract (Pizzarello, 2002). That examination reported an L-enantiomeric excess of $17.8 \%$; however, a brief mention in a book chapter (Pizzarello et al., 2006) suggested that analysis of a second sample did not show any statistically significant enantiomeric excess and that the discrepancy was the result of terrestrial contamination or sample heterogeneity.

Table 3 compares the enantiomeric ratios of $\mathrm{D}-$ and L-isovaline with those of $R$ - and $S$-sec-butylamine. ${ }^{1}$ Given the previously reported variability observed in the organic composition in different fragments of the Murchison meteorites (Cronin and Pizzarello, 1983; Krishnamurthy et al., 1992; Pizzarello et al., 2003), we used a portion of the same meteorite fragment to extract isovaline. Our analyses of indicate that both the free and total meteoritic sec-butylamine are racemic, while the free and total isovaline contain significant enrichments of the L-enantiomer (Table 3). Isovaline in both the free and total amino acid extracts showed significant enrichments of the L-enantiomer, with enantiomeric excesses of $9.6 \%$ (free) and $9.8 \%$ (total) respectively. These excesses are in agreement with previous analyses,

\footnotetext{
${ }^{1}$ While $R$ - and $S$-notations are more chemically correct, the amino acid literature uses D- and L-notations based on analogy with glyceraldehyde; thus, for consistency to the nomenclature used on previous meteoritic amino acid studies, we equate $R$ - to L- and $S$ - to D-based on their structures.
}

Table 3

Enantiomeric ratios for sec-butylamine $(R / S)$ and isovaline $(\mathrm{D} / \mathrm{L})$ extracted from the Murchison CM2 carbonaceous chondrite.

\begin{tabular}{lll}
\hline Compound & \multicolumn{2}{l}{ Enantiomeric ratios $^{\mathrm{a}}$} \\
\cline { 2 - 3 } & Free & Total \\
\hline sec-Butylamine $(R / S)^{\mathrm{b}}$ & $1.00 \pm 0.01$ & $0.99 \pm 0.01$ \\
${\text { Isovaline }(\mathrm{D} / \mathrm{L})^{\mathrm{c}}}^{\text {3-Methyl-2-butylamine }(R / S)^{\mathrm{b}}}$ & $0.83 \pm 0.01$ & $0.82 \pm 0.02$ \\
${\text { sec-Pentylamine }(R / S)^{\mathrm{b}}}^{\mathrm{b}}$ & $0.90 \pm 0.03$ & $0.91 \pm 0.02$ \\
\hline
\end{tabular}

${ }^{a}$ Ratios have been corrected against injections of racemic standards to account for instrument response.

b Enantiomeric ratios and standard deviations are based on three separate measurements from single ion gas chromatograms extracted at $\mathrm{m} / \mathrm{z}=166.0 \pm 0.5$ for sec-butylamine and at $\mathrm{m} /$ $z=236-238 \pm 0.5$ for sec-pentylamine and 3-methyl-2-butylamine.

${ }^{\mathrm{c}}$ Isovaline was extracted from a piece of inner Murchison. Enantiomeric ratios and standard deviations are based on three separate measurements from single ion LC-FD/ToF-MS chromatograms extracted at $m / z=379.13 \pm 0.015$.

which reported varying degrees of L-isovaline enantiomeric excess up to $18 \%$ in the Murchison meteorite (Cronin and Pizzarello, 1997; Pizzarello et al., 2003; Glavin and Dworkin, 2009).

The observations of different enantiomeric compositions but similar distribution of the constitutional isomers and carbon isotopic ratios for sec-butylamine and isovaline can constrain the synthetic relationship between these two compounds. While the location and processes that lead to the enantioenrichment of isovaline remain unclear, our data indicate that those processes did not create similar enantiomeric enrichments of sec-butylamine. Two primordial 
environments can be envisioned for the formation of these compounds: cold interstellar regions and the Murchison parent body. The rate of racemization of sec-butylamine and isovaline under radiation in cold interstellar simulated conditions remains to be determined. However, $\alpha$-dialkyl amino acids are highly resistant to racemization (Pollock et al., 1975); similarly, the low acidity of the $\alpha$-hydrogen present in sec-butylamine also makes this compound resistant to racemization $(\mathrm{pKa}=40$; Bordwell et al., 1981) through parent body processes. Thus, any large primordial enantiomeric excess occurring before their incorporation to the parent body should have been observable from our sample.

The similarity in the distribution of their constitutional isomers and ${ }^{13} \mathrm{C}$ isotopic composition of sec-butylamine and isovaline suggests that these compounds may have formed from the same carbon pool and precursors, and that the enantiomeric imbalance found in isovaline developed through yet-to-be-discovered mechanisms that did not have an effect on sec-butylamine. Such common precursors could include sec-butylimine and its hydrolysis product methyl ethyl ketone. The achiral imine or ketone may undergo a cyanohydrin (Strecker-type) reaction (Peltzer and Bada, 1978; Peltzer et al., 1984) to form isovaline or could undergo hydrogenation (from the imine) or reductive amination (from the ketone) to form sec-butylamine. It is plausible that a small enantiomeric excess (within our experimental error) could be introduced to either compound during these formation processes if the reactions are driven by circularly polarized light as previously shown for the synthesis of D- and L-alanine (Takano et al., 2007; De Marcellus et al., 2011). This small excess could then be amplified through parent-body processes (Glavin et al., 2012) that affected the isovaline but not the sec-butylamine, leading to the observed enantiomeric compositions. Further experiments are needed to prove if symmetry breaking can occur when synthesizing sec-butylamine and isovaline from achiral reagents and to examine potential amplification mechanisms.

There may also be direct synthetic connections between the sec-butylamine and isovaline through either the loss or addition of a $\mathrm{CO}_{2}$ group. The similarity in ${ }^{13} \mathrm{C}$ isotopic composition of the two compounds suggests that the $\delta^{13} \mathrm{C}$ value of the carbon in that $\mathrm{CO}_{2}$ is not significantly different from the $\delta^{13} \mathrm{C}$ value of the other four carbons. The formation of sec-butylamine through the decarboxylation of isovaline has been previously proposed (Pizzarello et al., 1994). Although decarboxylation experiments for isovaline under cosmochemical conditions have yet to be reported, bond breaking induced by radiation should result in higher degrees of decomposition than racemization, as exemplified by the low degree of racemization found after photolysis of L-alanine (Ehrenfreund et al., 2001b). Furthermore, it is likely that decarboxylation would lead to the loss of any enantiomeric excess present in the amino acid parent molecule because of the formation of a prochiral ion intermediate. It is unclear, however, how prominent this decomposition reaction may have been on the asteroid parent body, given the low temperature of aqueous alteration (maximum $80^{\circ} \mathrm{C}$; Brearley, 2006; Aponte et al., 2011), and the shielding offered by the parent body against cosmic radiation. Therefore, only a minor portion of the sec-butylamine found in Murchison may derive from the decarboxylation of isovaline.

A second potential synthetic relationship between secbutylamine and isovaline is the formation of isovaline through the addition of carbon dioxide $\left(\mathrm{CO}_{2}\right)$ to the amine (Hudson et al., 2009). Gas-grain phase reactions happening on the surface of cold grains coated with ices via ions and radical species using achiral precursors will result in racemic products (Cronin and Chang, 1993; Herbst, 1995; Bernstein et al., 2002; Muñoz Caro et al., 2002). When such reactions are driven by ultraviolet circularly polarized light, however, small enantiomeric excesses may be produced (Takano et al., 2007; De Marcellus et al. 2011), although no experiments have yet examined the synthesis of isovaline from racemic sec-butylamine under these conditions. The addition of $\mathrm{CO}_{2}$ (or other acid forming species such as a nitrile group) to sec-butylamine after incorporation into the parent body seems unlikely given the low acidity of the amine $\alpha$-hydrogen and the shielding of these molecules from cosmic radiation. Further investigations on the isotopic and enantiomeric composition of sec-butylamine and isovaline present in carbonaceous chondrites of different petrologic types are needed to understand the influence of thermal and aqueous processes on the origins and evolution of these chiral molecules.

\section{CONCLUSIONS}

The purpose of this study was threefold: (a) to analyze the abundance and distribution of free and bound meteoritic amines; (b) to determine the enantiomeric composition of sec-butylamine and compare to that of isovaline in a single meteorite sample; and (c) to evaluate the ${ }^{13} \mathrm{C}$ isotopic composition of aliphatic amines present in the Murchison meteorite for the first time. This compilation of data, combined with previous analyses of meteoritic amino acids, allowed us to evaluate the origins and synthetic mechanisms of these compounds under cosmochemical conditions:

- We observed trends of abundance and structural diversity similar to those previously reported for meteoritic amino acids and carboxylic acids, which may be indicative of the homogeneous synthetic origins of organic compounds in the Solar System.

- We found large ${ }^{13} \mathrm{C}$ enrichments of methylamine and ethylamine relative to $\mathrm{C}_{3}$ and $\mathrm{C}_{4}$ amines, as well as between $\mathrm{C}_{1}$ and $\mathrm{C}_{3}$ linear amines and their structurally analogous amino acids, while no significant ${ }^{13} \mathrm{C}$ isotopic differences were observed for branched $\mathrm{C}_{3}-\mathrm{C}_{4}$ amines and their structural amino acid analogs. These results may be consistent with the chain elongation mechanism under kinetic control previously proposed for other meteoritic aliphatic organic compounds.

- sec-Butylamine and isovaline exhibited similar $\delta^{13} \mathrm{C}$ values; in contrast, sec-butylamine was racemic but L-isovaline extracted from the same Murchison piece showed $9.7 \%$ ee. These results suggest that sec-butylamine and isovaline may share common synthetic origins, but that processes that led to enantiomeric excess in the amino acid did not affect the amine. 
Further analyses of the isotopic and enantiomeric composition of aliphatic amines and their structurally analogous amino acids present in various different types of carbonaceous chondrites may shed light on the primordial synthesis and development of these compounds.

\section{ACKNOWLEDGMENTS}

The authors would like to thank H. McLain for assistance in the data analysis of the amino acids study, T. McCoy, L. Welzenbach, and the Smithsonian National Museum of Natural History Division of Meteorites for providing the meteorite sample used in this study, and D.P. Glavin for his helpful comments during preparation of the manuscript. J.C.A. acknowledges support from the NASA Postdoctoral Program at the Goddard Space Flight Center, administered by Oak Ridge Associated Universities and the NASA Astrobiology Institute through a contract with NASA. This research was supported by the NASA Astrobiology Institute and the Goddard Center for Astrobiology, NASA's Cosmochemistry Program, and a grant from the Simons Foundation (SCOL award 302497). The authors acknowledge Associate Editor George Cooper and two anonymous reviewers for their helpful comments on the manuscript.

\section{APPENDIX A. SUPPLEMENTARY DATA}

Supplementary data associated with this article can be found, in the online version, at http://dx.doi.org/10.1016/ j.gca.2014.06.035.

\section{REFERENCES}

Anders E. (1989) Pre-biotic organic matter from comets and asteroids. Nature 342, 255-257.

Aponte J. C., Alexandre M. R., Wang Y., Brearley A. J., Alexander C. O'D. and Huang Y. (2011) Effects of secondary alteration on the composition of free and IOM-derived monocarboxylic acids in carbonaceous chondrites. Geochim. Cosmochim. Acta 75, 2309-2323.

Aponte J. C., Tarozo R., Alexandre M. R., Alexander C. M. O’D., Charnley S. B., Hallmann C., Summons R. and Huang Y. (2014) Chirality of meteoritic free and IOM-derived monocarboxylic acids and implications for prebiotic organic synthesis. Geochim. Cosmochim. Acta 131, 1-12.

Bernstein M. P., Dworkin J. P., Sandford S. A., Cooper G. W. and Allamandola L. J. (2002) Racemic amino acids from the ultraviolet photolysis of interstellar ice analogues. Nature 416, 401-403.

Bonner W. A. (1991) The origin and amplification of biomolecular chirality. Orig. Life Evol. Biosph. 21, 59-111.

Bordwell F. G., Drucker G. E. and Fried H. E. (1981) Acidities of carbon and nitrogen acids: the aromaticity of the cyclopentadienyl anion. J. Org. Chem. 46, 632-635.

Brearley A. J. (2006) The action of water. In Meteorites and the Early Solar System II (eds. D. S. Lauretta, L. A. Leshin and H. Y. McSween). University of Arizona Press, Tucson, AZ, pp. 587-624.

Burton A. S., Elsila J. E., Callahan M. P., Martin M. G., Glavin D. P., Johnson N. M. and Dworkin J. P. (2012) A propensity for $n$ $\omega$-amino acids in thermally altered Antarctic meteorites. Meteorit. Planet. Sci. 47, 374-386.

Burton A. S., Elsila J. E., Hein J. E., Glavin D. P. and Dworkin J. P. (2013) Extraterrestrial amino acids identified in metal-rich
$\mathrm{CH}$ and $\mathrm{CB}$ carbonaceous chondrites from Antarctica. Meteorit. Planet. Sci. 48, 390-402.

Callahan M. P., Smith K. E., Cleaves, II, H. J., Ruzicka J., Stern J. C., Glavin D. P., House C. H. and Dworkin J. P. (2011) Carbonaceous meteorites contain a wide range of extraterrestrial nucleobases. Proc. Natl. Acad. Sci. U.S.A. 108, 1399513998.

Charnley S. B., Ehrenfreund P., Millar T. J., Boogert A. C. A., Markwick A. J., Butner H. M., Ruiterkamp R. and Rodgers S. D. (2004) Observational tests for grain chemistry: posterior isotopic labelling. Mon. Not. R. Astron. Soc. 347, 157-162.

Chyba C. and Sagan C. (1992) Endogenous production, exogenous delivery and impact-shock synthesis of organic molecules: an inventory for the origins of life. Nature 355, 125-132.

Cooper G., Kimmich N., Belisle W., Sarinana J., Brabham K. and Garrel L. (2001) Carbonaceous meteorites as a source of sugarrelated organic compounds for the early Earth. Nature 414, 879-883.

Cronin J. R. and Pizzarello S. (1983) Amino acids in meteorites. Adv. Space Res. 3, 5-18.

Cronin J. R. and Pizzarello S. (1986) Amino acids of the Murchison meteorite. III. Seven carbon acyclic primary $\alpha$-amino alkanoic acids. Geochim. Cosmochim. Acta 50, 2419-2427.

Cronin J. R. and Chang S. (1993) Organic matter in meteorites: molecular and isotopic analyses of the Murchison meteorite. In The Chemistry of Life's Origins (eds. J. M. Greenberg, C. X. Mendoza-Gómez and V. Pirronello). Kluwer Academic Publishers, pp. 209-258.

Cronin J. R. and Pizzarello S. (1997) Enantiomeric excesses in meteoritic amino acids. Science 275, 951-955.

Cronin J. R., Pizzarello S. and Yuen G. U. (1985) Amino acids of the Murchison meteorite: II. Five carbon acyclic primary $\beta-, \gamma-$, and $\delta$-amino alkanoic acids. Geochim. Cosmochim. Acta 49, 2259-2265

De Leuw S., Rubin A. E. and Wasson J. T. (2010) Carbonates in $\mathrm{CM}$ chondrites: Complex formational histories and comparison to carbonates in CI chondrites. Meteorit. Planet. Sci. 45, 513530.

De Marcellus P., Meinert C., Nuevo M., Filippi J.-J., Danger G., Deboffle D., Nahon L., D'Hendecourt L., Le S. and Meierhenrich U. J. (2011) Non-racemic amino acid production by ultraviolet irradiation of achiral interstellar ice analogs with circularly polarized light. Astrophys. J. Lett. 727, L27.

Docherty G., Jones V. and Evershed R. P. (2001) Practical and theoretical considerations in the gas chromatography/combustion/isotope ratio mass spectrometry $\delta^{13} \mathrm{C}$ analysis of small polyfunctional compounds. Rapid Commun. Mass Spectrom. 15, 730-738.

Ehrenfreund P., Glavin D. P., Botta O., Cooper G. and Bada J. L. (2001a) Extraterrestrial amino acids in Orgueil and Ivuna: tracing the parent body of CI type carbonaceous chondrites. Proc. Natl. Acad. Sci. U.S.A. 98, 2138-2141.

Ehrenfreund P., Bernstein M. P., Dworkin J. P., Sandford S. A. and Allamandola L. J. (2001b) The photostability of amino acids in space. Astrophys. J. 550, L95-L99.

Elsila J. E., Glavin D. P. and Dworkin J. P. (2009) Cometary glycine detected in samples returned by Stardust. Meteorit. Planet. Sci. 44, 1323-1330.

Elsila J. E., Charnley S. B., Burton A. S., Glavin D. P. and Dworkin J. P. (2012) Compound-specific carbon, nitrogen, and hydrogen isotopic ratios for amino acids in $\mathrm{CM}$ and $\mathrm{CR}$ chondrites and their use in evaluating potential formation pathways. Meteorit. Planet. Sci. 47, 1517-1536.

Engel M. H. and Macko S. A. (1997) Isotopic evidence for extraterrestrial non-racemic amino acids in the Murchison meteorite. Nature 389, 265-268. 
Engel M. H., Macko S. A. and Silfer J. A. (1990) Carbon isotope composition of individual amino acids in the Murchison meteorite. Nature 348, 47-49.

Epstein S., Krishnamurthy R. V., Cronin J. R., Pizzarello S. and Yuen G. U. (1987) Unusual stable isotope ratios in amino-acid and carboxylic-acid extracts from the Murchison meteorite. Nature 326, 477-479.

Glavin D. P. and Dworkin J. P. (2009) Enrichment of the amino acid L-isovaline by aqueous alteration on $\mathrm{CI}$ and $\mathrm{CM}$ meteorite parent bodies. Proc. Natl. Acad. Sci. U.S.A. 106, 5487-5492.

Glavin D. P., Dworkin J. P., Aubrey A., Botta O., Doty, III, J. H., Martins Z. and Bada J. L. (2006) Amino acid analyses of Antarctic CM2 meteorites using liquid chromatography-time of flight-mass spectrometry. Meteorit. Planet. Sci. 41, 889-902.

Glavin D. P., Dworkin J. P. and Sandford S. A. (2008) Detection of cometary amines in samples returned by Stardust. Meteorit. Planet. Sci. 43, 399-413.

Glavin D. P., Callahan M. P., Dworkin J. P. and Elsila J. E. (2011) The effects of parent body processes on amino acids in carbonaceous chondrites. Meteorit. Planet. Sci. 45, 19481972.

Glavin D. P., Elsila J. E., Burton A. S., Callahan M. P., Dworkin J. P., Hilts R. W. and Herd C. D. K. (2012) Unusual nonterrestrial L-proteinogenic amino acid excesses in the Tagish Lake meteorite. Meteorit. Planet. Sci. 47, 1347-1364.

Greenberg J. M. (1996) Chirality in interstellar dust and in comets: life from dead stars. In Proceedings 379 of the Santa Monica Workshop on the Physical Origin of Homochirality in Life, February 1996. Santa Monica, California, American Institute of Physics, New York. pp. 185-210.

Herbst E. (1995) Chemistry in the interstellar medium. Annu. Rev. Phys. Chem. 46, 27-53.

Huang Y., Wang Y., Alexandre M. R., Lee T., Rose-Petruck C., Fuller M. and Pizzarello S. (2005) Molecular and compoundspecific isotopic characterization of monocarboxylic acids in carbonaceous meteorites. Geochim. Cosmochim. Acta 69, 10731084.

Hudson R. L., Lewis A. S., Moore M. H., Dworkin J. P. and Martin M. P. (2009) Enigmatic isovaline: investigating the stability, racemization, and formation of a non-biological meteoritic amino acid. ASP Conf. Ser. 420, 157-162.

Klussman M., Iwamura H., Mathew S. P., Wells, Jr., D. H., Pandya U., Armstrong A. and Blackmond D. G. (2006) Thermodynamic control of asymmetric amplification in amino acid catalysis. Nature 441, 621-623.

Krishnamurthy R. V., Epstein S., Cronin J. R., Pizzarello S. and Yuen G. U. (1992) Isotopic and molecular analyses of hydrocarbons and monocarboxylic acids of the Murchison meteorite. Geochim. Cosmochim. Acta 56, 4045-4058.

Kvenvolden K., Lawless J., Pering K., Peterson E., Flores J., Ponnamperuma C., Kaplan I. R. and Moore C. (1970) Evidence for extraterrestrial amino-acids and hydrocarbons in the Murchison meteorite. Nature 228, 923-926.

Jungclaus G. A., Yuen G. U., Moore C. B. and Lawless J. G. (1976) Evidence for the presence of low molecular weight alcohols and carbonyl compounds in the Murchison meteorite. Meteoritics 11, 231-237.

Larsen T., Taylor D. L., Leigh M. B. and O'Brien D. M. (2009) Stable isotope fingerprinting: a novel method for identifying plant, fungal, or bacterial origins of amino acids. Ecology $\mathbf{9 0}$, 3526-3535.

LeVasseur N. L., Zhu H.-J., Markowitz J. S., DeVane C. L. and Patrick K. S. (2008) Enantiospecific gas chromatographic-mass spectrometric analysis of urinary methylphenidate: implications for phenotyping. J. Chromatogr. B 862, 140-149.
Martins Z., Alexander C. M. O. D., Orzechowska G. E., Fogel M. L. and Ehrenfreund P. (2007) Indigenous amino acids in primitive CR meteorites. Meteorit. Planet. Sci. 42, 2125-2136.

Martins Z., Botta O., Fogel M. L., Sephton M. A., Glavin D. P., Watson J. S., Dworkin J. P., Schwartz A. W. and Ehrenfreund P. (2008) Extraterrestrial nucleobases in the Murchison meteorite. Earth Planet. Sci. Lett. 270, 130-136.

Meierhenrich U. J., Muñoz-Caro G. M., Bredehöft J. H., Jessberger E. K. and Thiemann W. H.-P. (2004) Identification of diamino acids in the Murchison meteorite. Proc. Natl. Acad. Sci. U.S.A. 101, 9182-9186.

Meinert C., Hoffmann S. V., Cassam-Chenaï P., Evans A. C., Giri C., Nahon L. and Meierhenrich U. J. (2014) Photonenergycontrolled symmetry breaking with circularly polarized light. Angew. Chem. Int. Ed. 53, 210-214.

Muñoz Caro G. M., Meierhenrich U. J., Schutte W. A., Barbier B., Arcones Segovia A., Rosenbauer H., Thiemann W. H.-P., Brack A. and Greenberg J. M. (2002) Amino acids from ultraviolet irradiation of interstellar ice analogues. Nature 416, 403-406.

Modica P., Meinert C., de Marcellus P., Nahon L., Meierhenrich U. J. and d'Hendecourt L. L. S. (2014) Enantiomeric excesses induced in amino acids by ultraviolet circularly polarized light irradiation of extraterrestrial ice analogs: A possible source of asymmetry for prebiotic chemistry. Astrpphys. J. 788, 79 (11 pp).

Mohr S., Weiß J. A., Spreitz J. and Schmid M. G. (2012) Chiral separation of new cathinone- and amphetamine-related designer drugs by gas chromatography-mass spectrometry using trifluoroacetyl-L-prolyl chloride as chiral derivatization reagent. J. Chromatogr. A 1269, 352-359.

Peltzer E. T. and Bada J. L. (1978) Alpha-hydroxycarboxylic acids in the Murchison meteorite. Nature 272, 443-444.

Peltzer E. T., Bada J. L., Schlesinger G. and Miller S. L. (1984) The chemical conditions on the parent body of the Murchison meteorite: some conclusions based on amino, hydroxy and dicarboxylic acids. Adv. Space Res. 4, 69-74.

Pizzarello S. (2002) The chiral amines of the Murchison meteorite: a preliminary characterization (abstract). Lunar Planet. Sci. XXXIII. Lunar Planet. Inst., Houston. (CD-ROM) \#1233 (abstr.).

Pizzarello S. and Cronin J. R. (2000) Non-racemic amino acids in the Murray and Murchison meteorites. Geochim. Cosmochim. Acta 64, 329-338.

Pizzarello S. and Cooper G. W. (2001) Molecular and chiral analyses of some protein amino acid derivatives in the Murchison and Murray meteorites. Meteorit. Planet. Sci. 36, 897-909.

Pizzarello S. and Holmes W. (2009) Nitrogen-containing compounds in two CR2 meteorites: $15 \mathrm{~N}$ composition, molecular distribution and precursor molecules. Geochim. Cosmochim. Acta 73, 2150-2162.

Pizzarello S. and Shock E. (2010) The organic composition of carbonaceous meteorites: the evolutionary story ahead of biochemistry. Cold Spring Harb. Perspect. Biol. 2, a002105.

Pizzarello S. and Groy T. (2011) Molecular asymmetry in extraterrestrial organic chemistry: an analytical perspective. Geochim. Cosmochim. Acta 75, 645-656.

Pizzarello S., Krishnamurthy R. V., Epstein S. and Cronin J. R. (1991) Isotopic analyses of amino acids from the Murchison meteorite. Geochim. Cosmochim. Acta 55, 905-910.

Pizzarello S., Feng X., Epstein S. and Cronin J. R. (1994) Isotopic analyses of nitrogenous compounds from the Murchison meteorite: ammonia, amines, amino acids, and polar hydrocarbons. Geochim. Cosmochim. Acta 58, 5579-5587. 
Pizzarello S., Zolensky M. and Turk K. A. (2003) Non racemic isovaline in the Murchison meteorite: chiral distribution and mineral association. Geochim. Cosmochim. Acta 67, 1589-1595.

Pizzarello S., Huang Y. and Fuller M. (2004) The carbon isotopic distribution of Murchison amino acids. Geochim. Cosmochim. Acta 68, 4963-4969.

Pizzarello S., Cooper G. W. and Flynn G. J. (2006) The nature and distribution of the organic material in carbonaceous chondrites and interplanetary dust particles. In Meteorites and the Early Solar System II (eds. D. S. Lauretta and H. Y. McSween). University of Arizona Press, Tucson, AZ, pp. 625-651.

Pizzarello S., Huang Y. and Alexandre M. R. (2008) Molecular asymmetry in extraterrestrial chemistry: insights from a pristine meteorite. Proc. Natl. Acad. Sci. U.S.A. 105, 3700-3704.

Pizzarello S., Schrader D. L., Monroe A. A. and Lauretta D. S. (2012) Large enantiomeric excesses in primitive meteorites and the diverse effects of water in cosmochemical evolution. Proc. Natl. Acad. Sci. U.S.A. 109, 11949-11954.

Pollock G. E., Cheng C.-N., Cronin S. E. and Kvenvolden K. A. (1975) Stereoisomers of isovaline in the Murchison meteorite. Geochim. Cosmochim. Acta 39, 1571-1573.

Sandford S. A., Aléon J., Alexander C. M. O’D., Araki T., Bajt S., Baratta G. A., Borg J., Brucato J. R., Burchell M. J., Busemann H., Butterworth A., Clemett S. J., Cody G., Colangeli L., Cooper G., D'Hendecourt L., Djouadi Z., Dworkin J. P., Ferrini G., Fleckenstein H., Flynn G. J., Franchi I. A., Fries M., Gilles M. K., Glavin D. P., Gounelle M., Grossemy F., Jacobsen C., Keller L. P., Kilcoyne D., Leitner J., Matrajt G., Meibom A., Mennella V., Mostefaoui S., Nittler L. R., Palumbo M. E., Robert F., Rotundi A., Snead C. J., Spender M. K., Steele A., Stephan T., Tyliszczak T., Westphal A. J., Wirick S., Wopenka B., Yabuta H., Zare R. N. and Zolensky M. (2006) Organics captured from comet Wild 2 by the Stardust spacecraft. Science 314, 1720-1724.

Schmitt-Kopplin P., Gabelica Z., Gougeon R. D., Fekete A., Kanawati B., Harir M., Gebefuegi I., Eckel G. and Hertkorn
N. (2010) High molecular diversity of extraterrestrial organic matter in Murchison meteorite revealed 40 years after its fall. Proc. Natl. Acad. Sci. U.S.A. 107, 2763-2768.

Scott J. H., O'Brien D. M., Emerson D., Sun H., McDonald G. D., Salgado A. and Fogel M. L. (2006) An examination of the carbon isotope effects associated with amino acid biosynthesis. Astrobiology 6, 867-880.

Soai K., Shibata T., Morioka H. and Choji K. (1995) Asymmetric autocatalysis and amplification of enantiomeric excess of a chiral molecule. Nature 378, 767-768.

Takano Y., Takahashi J., Kaneko T., Marumo K. and Kobayashi K. (2007) Asymmetric synthesis of amino acid precursors in interstellar complex organics by circularly polarized light. Earth Planet. Sci. Lett. 254, 106-114.

Theule P., Borget F., Mispelaer F., Danger G., Duvernay F., Guillemin J. C. and Chiavassa T. (2011) Hydrogenation of solid hydrogen cyanide $\mathrm{HCN}$ and methanimine $\mathrm{CH}_{2} \mathrm{NH}$ at low temperature. Astron. Astrophys. 534, A64.

Tielens A. G. G. M. and Hagen W. (1982) Model calculations of the molecular composition of interstellar grains mantles. Astron. Astrophys. 114, 245-260.

Van Bocxlaer J. F., Lambert W. E., Thienpont L. and DeLeenheer A. P. (1997) Quantitative determination of amphetamine and $\alpha$ phenylethylamine enantiomers in judicial samples using capillary gas chromatography. J. Analyt. Toxicol. 21, 5-11.

Yuen G. U. and Kvenvolden K. A. (1973) Monocarboxylic acids in Murray and Murchison carbonaceous meteorites. Nature 246, 301-303.

Yuen G., Blair N., Des Marais D. J. and Chang S. (1984) Carbon isotopic composition of individual, low molecular weight hydrocarbons and monocarboxylic acids from the Murchison meteorite. Nature 307, 252-254.

Associate editor: George Cooper 